\title{
Aprendizagem da Anatomia Humana: contribuição de um roteiro teórico-prático
}

\section{Human Anatomy learning: contribution of a theoretical-practical script}

\author{
Rafael Galisa de Oliveira ${ }^{1}$ \\ Carla Patricia Bejo Wolkers ${ }^{2}$
}

\section{RESUMO}

0 processo de ensino-aprendizagem da anatomia humana é complexo e tem sofrido constantes modificações. Devido as dificuldades que perpassam este processo, métodos inovadores de aprendizagem são necessários para solucionar essa problemática. À vista disso, o presente trabalho avaliou a efetividade de um roteiro teórico-prático no processo de ensino-aprendizagem desta disciplina, no curso de ciências biológicas. 0 roteiro contribuiu com o processo de ensino-aprendizagem da anatomia, despertou o interesse dos estudantes pela monitoria e foi considerado uma ferramenta complementar ao uso do cadáver. Embora os participantes tenham tido maior dificuldade com a nomenclatura anatômica, o roteiro teve maior contribuição com a aprendizagem da classificação. Esta ferramenta é auxiliar na construção do conhecimento dos discentes sobre esta ciência.

\section{PALAVRAS-CHAVE}

Aprendizagem; Cadáver; Ciências Biológicas; Ensino; Saúde.

\footnotetext{
${ }^{1}$ Acadêmico da Universidade Federal de Uberlândia.

${ }^{2}$ Docente da Universidade Federal de Uberlândia.
} 


\section{ABSTRACT}

The teaching-learning process of human anatomy is complex and has undergone frequent modifications. Despite the difficulties that pass through this process, innovative learning methods are needed to solve these problems Therefore, the present study evaluated the effectiveness of a theoretical-practical scripts in the anatomy teaching-learning process, in Biological Sciences undergraduation course. The script contributed with the anatomy teaching-learning process, increased the students interest in monitoring and was considered a complementary tool to the use of cadaver. Although the participnts have greater difficulty with the anatomical nomenclature, the script had greater contribuition with the classification learning. This tool is auxiliary in construction of scientific of students about this science.

\section{KEYWORDS}

Learning; Cadaver; Biological Sciences; Teaching; Health. 


\section{INTRODUÇÃO}

A Anatomia Humana é um componente curricular presente nos cursos da área de ciências da saúde (KRUSE, 2004; SOCIEDADE BRASILEIRA DE ANATOMIA SBA, [2012])e ciências biológicas (SIQUEIRA NETO; FERREIRA, 2001). Seu processo de aprendizagem é complexo e demorado, mas essencial para o conhecimento das estruturas do corpo humano e de suas relações (TORTORA; NIELSE, 2013).

Ao longo dos anos, o ensino da anatomia tem sofrido uma série de influências e modificações. Embora o cadáver ainda seja um dos principais alvos de estudo (GHOSH, 2017; JOHNSON; CHARCHANTI; TROUPIS 2012), outros métodos inovadores de aprendizagem têm sido empregados na busca da superação das dificuldades apresentadas pelos discentes, como o uso de atlas eletrônico interativo (IeA) e animaçõeson-linesobre partes docorpo humano, a fim de melhorar o processo de aprendizagem (GUY et al., 2015; LONE et al., 2018; PIAZZA; CHASSOT, 2012; RICH; GUY, 2013). Além disso, os livros e atlas didáticos são frequentemente atualizadospor meio da utilização de imagens reais, figuras e linguagem mais clara, visando facilitar o processo de construção do conhecimento e melhor formação dos estudantes (WATANABE, 2009; NETTER, 2011; MOORE; AGUR, 2004; TORTORA; NIELSEN, 2013).

De fato, o emprego de metodologias que permitem o uso de várias funções cognitivas, como ver, ouvir, interagir e executar (BRAZ, 2009), além do estímulo ao estudo em pares (KRYCH et al., 2005), e a diversificação dos materiais didáticos(FALAVIGNA, 2009), estão associados a melhorias nas habilidades de comunicação e aprendizagem dos estudantes. Além disso, estudos sugerem que mudanças na dinâmica das aulas, com a implementação de metodologias de ensino descontraídas e atrativas fazem com que os estudantes tenham maior interesse na disciplina, levando à redução na memorização, facilitando a aprendizagem (PIAZZA; CHASSOT, 2012), o que reforça a necessidade de implementação de práticas didáticas que fogem do ensino tradicional para o aprendizado de disciplinas como a anatomia. Ações desta natureza permitem uma transposição didática efetiva (MASETTO, 1997), fazendo com que a atividade pedagógica seja estruturada a partir de uma nova abordagem para a construção do conhecimento, unindo os diversos saberes do professor com o objetivo de permitir que os estudantes compreendam e se apropriem do conteúdo apresentado.

Na universidade federal de Uberlândia - campus Pontal, onde o estudo foi realizado, o ensino da anatomia humana para os cursos de Ciências Biológicas Licenciatura e Bacharelado dá-se por meio de aulas teóricas predominantemente expositivas em sala de aula, seguidas de aulas práticas em laboratório, utilizando atlas, livros e peças anatômicas sintéticas e naturais. A dificuldade com a nomenclatura e classificação anatômicas e a falta de tempo para ministrar todo conteúdo previsto, além do forte odor de formol exalado pelas peças anatômicas cadavéricas e o alto grau de degradação dessas, são as principais adversidades encontradas no processo de ensino-aprendizagem da disciplina nos cursos dessa instituição. Nesse contexto, o objetivo deste estudo foi confeccionar e integrar um roteiro teórico-prático às aulas de anatomia humana e avaliar a efetividade desta ferramenta no processo de ensino-aprendizagem dessa disciplina, visto que, a avaliação e o usodessa ferramenta no estudo prático da anatomia humana são escassos na literaturaea aplicação de métodos inovadores nesta área têm revelado resultados positivos naaprendizagem dos alunos (BRUECKNER; MACPHERSON, 2004; FRUHSTORFER et al., 2011; GLITTENBERG; BINDER, 2006; KHARB; SAMANTA, 2016; LONE et al., 2018; NASCIMENTO et al., 2014; PEREIRA et al., 2007).

\section{MATERIAIS E MÉTODOS}

O estudo foi realizado comdiscentes matriculados nos cursos de Ciências Biológicas, GrauBacharelado e Grau Licenciaturada Universidade Federal de Uberlândia - campus Pontal, no período de 2015 a 2017. No curso de Ciências Biológicas o componente curricular Anatomia Humana é ministrado por um docente e conta com o auxílio de 3 a 5 monitores que atendem em horários diversos. A disciplina possui carga horária de 75 horas para o Grau Bacharelado (sendo 30 horas práticas) e 45 horas para o Grau Licenciatura (sendo 15 horas práticas).

0 estudo foi realizado em 4 etapas: (1) levantamento das dúvidas e dificuldades dos estudantes na aprendizagem da anatomia humana, (2) seleção do conteúdo e produção do roteiro teórico-prático, (3) aplicação do 
roteiro teórico-prático e, (4) avaliação do roteiro teórico-prático no processo de ensino-aprendizagem.

\section{Levantamento das dúvidas e dificuldades}

O levantamento das principais dificuldades dos alunos do Curso de Ciências Biológicas matriculados no componente curricular Anatomia Humana foi realizado no primeiro (bacharelado) e segundo (licenciatura) semestre letivo de 2016. 0 processo deu-se por meio de uma análise qualitativa durante as monitorias e aulas práticas da disciplina, na qual efetuou-se observações e anotações das dúvidas e dificuldades expressadas pelos estudantessobre cada sistema do corpo humano. Posteriormente, os dados coletados foram tabulados usando o Microsoft Excel@ e as principais dificuldades referidas foram identificadas e classificadas, e o nível de dificuldade em cada ponto avaliado foi classificado em BAIXO, MÉDIO e ALTO.

\section{Seleção do conteúdo e produção do roteiro}

Após a identificação e classificação das principais dúvidas e dificuldades apresentadas pelos discentes, foi realizada uma pesquisa bibliográfica nos principais livros da área de anatomia do acervo da Biblioteca SetorialItuiutaba na Universidade Federal de Uberlândia e no blog de Anatomia e Fisiologia Comparada (http:// biologiapontal.blogspot.com.br/) desta instituição. Em seguida,realizou-se a seleção do conteúdo que compôs o roteiro. Foram utilizados textos, figuras, campos para desenho e anotações, tabelas e estudos dirigidos com questões de múltipla escolha e discursivas.0 roteiro foi composto por 11 capítulos, sendo o primeiro uma introdução sobre o estudo da Anatomia Humana,e os demais sobre o sistema muscular (SM), articular (AS), esquelético(SE), cardiovascular (SC), respiratório (SR), genital masculino (SGm), genital feminino(SGf), urinário (SU), digestório (SD) e neural (SN).Cada capítulocontava com figurase pequenos textos explicativos trazendo os principais conceitos sobre o sistema em questão eambos continham lacunas vazias que poderiam ser preenchidas pelos alunos. Todas as bibliografias utilizadas na produção são de fácil acesso aos discentes, para otimizar a busca de informações necessárias ao preenchimento dessa ferramenta.

\section{Aplicação do roteiro}

0 roteiro foi aplicado ao curso de bacharelado durante o primeiro semestre de 2017 e ao curso de licenciatura durante o segundo semestre do mesmo ano. 0 preenchimento foi realizado com base nos conhecimentos construídos durante as aulas teóricas e práticas e com o auxílio das peças anatômicas (naturais e sintéticas), livros, atlas,internet e, em caso de dúvidas persistentes, do monitor e professor.

\section{Avaliação do roteiro}

A contribuição do roteiro ao processo de ensino-aprendizagem dos discentes foi avaliado por meio da comparação do desempenho dos discentes do ano de 2016 e de 2017 e mediante aplicação de um questionário semi-estrtuturado e auto-administrado aos discentes que utilizaram o roteiro. Neste, os participantes foram questionados quanto (1) aograu de dificuldade para entender a disciplina de Anatomia Humana, (2) se tinham maior dificuldade com a nomenclatura ou classificação das estruturas e sistemas, (3) contribuição do roteiro na aprendizagem da nomenclatura e classificação das estruturas e sistemas, (4)a frequência e o interesse nas monitorias para preencher o roteiro, (5) se o roteiro é uma ferramenta complementar ao usodo cadáver no processo de ensino-aprendizagem e (6) se o roteiro exerceu contribuição no processo de aprendizagem da anatomia humana.

\section{Análise Estatística}

A distribuição dos dados obtidos a partir do questionário semi-estrutuado foram analisadas por meio do teste Qui-Quadrado de Pearson, nível de significância de 5\%, por meio do programa estatístico SigmaStat32.

\section{Nota Ética}

O presente estudo foi aprovado junto ao comitê de ética para seres humanos da Universidade Federal de Uberlândia (UFU) (CEP/UFU) (CAAE: 53337115.1. 0000.5152). Os participantes da pesquisa assinaram um Termo Livre e Esclarecido e tiveram sua identidade preservada. 


\section{RESULTADOS E DISCUSSÃO}

No total, 69 discentes participaram da pesquisa, 39 pertencentes ao curso de Ciências Biológicas, Grau Bacharelado (14 do gênero masculino e 25 do gênero feminino) e 30 pertencentes ao curso de Ciências Biológicas, GrauLicenciatura (11 do gênero masculino, 18 do gênero feminino e 1 não declarado). A média da idade dos participantes do bacharelado foi 19,3 (min.=17; máx.=29) e da licenciatura 23,3 (min.=18; máx.=38).
O levantamento das dúvidas e dificuldades no curso de Ciências Biológicas, Grau Bacharelado (Tabela 1) mostra que os discentes apresentaram nível de dificuldade BAIXO para aprender a função de todos os sistemas e de suas diversas estruturas. Em relação ao aprendizado da nomenclatura, apresentaram nível dedificuldade MÉDIO para os sistemas respiratório, digestório e urinário enível de dificuldade ALTO para os demais sistemas.O nível de dificuldade para aprender a classificação foi BAIXO para os sistemas esquelético, muscular, genital masculino e nervoso enível de dificuldade MÉDIO para os demais sistemas.

Tabela 1 - Nível de dificuldade dos discentes do curso de bacharelado em relação a função, nomenclatura e classificação dos diferentes sistemas do corpo humano

\begin{tabular}{llll}
\hline Dificuldade & Função & Nomenclatura & Classificação \\
\hline Baixa & SA, SC, SD, SE, SGf, SGm, & & SE, SM, SGm, SN \\
\hline Média & SM, SN, SR, SU & SA, SC, SR, SD, SU, SGf \\
\hline Alta & - & SD, SR, SU & - \\
\hline
\end{tabular}

*SM: sistema muscular;AS: sistema articulr; SE: sistema esquelético;SC: sistema cardiovascular;SR: sistema respiratório;SGm: sistema genital masculino;SGf: sistema genital feminino;SU: sistema urinário;SD: sistema digestório; SN: sistema neural.

Fonte: elaborada pelos autores a partir dos dados da pesquisa.

No curso de Ciências Biológicas, Grau Licenciatura (Tabela 2), os discentes apresentaram nível de dificuldade BAXO para aprender a função de todos os sistemas e de suas diversas estruturas. Em relação ao aprendizado da nomenclatura, apresentaram nível de dificuldade MÉDIO para os sistemas digestório e urinário e nível de dificuldade ALTO para os demais sistemas. O nível de dificuldade para aprender a classificação foi BAIXO para o sistema esquelético, MÉDIO para os sistemas muscular, circulatório, respiratório, digestório, urinário, genital masculino e genital feminino, e nível de dificuldade ALTO para os sistemas articular e nervoso.

Tabela 2 - Nível de dificuldade dos discentes do curso de licenciatura em relação a função, nomenclatura e classificação dos diferentes sistemas do corpo humano

\begin{tabular}{llll}
\hline Dificuldade & Função & Nomenclatura & Classificação \\
\hline Baixa & $\begin{array}{l}\text { SA, SC, SD, SE, SGf, } \\
\text { SGm, SM, SN, SR, SU }\end{array}$ & SE \\
\hline Média & - & SD, SU & SM, SC, SR, SD, SU, SGm, SGf \\
\hline Alta & - & SA, SE,SM, SC, SGf, SGm, SN, SR & SA, SN \\
\hline
\end{tabular}

*SM: sistema muscular;AS: sistema articulr; SE: sistema esquelético;SC: sistema cardiovascular;SR: sistema respiratório;SGm: sistema genital masculino;SGf: sistema genital feminino;SU: sistema urinário;SD: sistema digestório; SN: sistema neural. Fonte: elaborada pelosautores a partir dos dados da pequisa. 
Os discentes do curso de licenciatura tiveram maior dificuldade no aprendizado da anatomia em relação ao bacharelado. Além disso, ao responderem o questionário, 53,6\% dos estudantes revelaram ter grau de dificuldade ALTO e 46,4\% BAIXO para aprender a disciplina, com diferença significativa entre os cursos (Qui-quadrado $=4,6183$, grau de liberdade $=1, p=0,03163$ ). A proporção de estudantes que consideraram o grau de dificuldade BAIXO, foi duas vezes maior na modalidade Bacharelado (60\%) em relação à Licenciatura (30\%). Não houve diferença significativa entre os gêneros masculino e feminino.

0 ensino-aprendizagem da anatomia humana é um processo complexo (ARRUDA; SOUSA, 2014; TORTORA; NIELSE, 2013) e essa diferença no grau de dificuldade entre as modalidades pode estar associada à diversos fatores presentes no dia-a-dia desses estudantes. 0 curso de licenciatura desta instituição constitui-se, em grande parte, de discentes mais velhos, que trabalham durante o dia e que estão há mais tempo afastados dos estudos. 0 exercício de outras atividades além da graduação inviabiliza a participação destes nas monitorias e diminui o tempo dedicado à disciplina. Somado a isso, muitos residem em cidades vizinhas à localidade da universidade, estando presentes, apenas,nos horários de aula. Situação semelhante a essa foi mostrada nos estudos de Salbego et al. (2015), realizado com os cursos de Ciências Biológicas e Enfermagem de uma universidade da Região Centro-Oeste do Estado do Rio Grande do Sul, em que foi apontado queestes fatores,juntamente comos compromissos e responsabilidades com a família, diminui o tempo de estudo e dificulta ainda mais o aprendizado dos discentes, além de colaborar para que a disciplina seja considerada uma das mais difíceis do início da graduação.

A aprendizagem da anatomia, muitas vezes, se dá pelo processo de memorização e fixação de conteúdo, ignorando o papel ativo do aluno na construção do conhecimento. Além disso, os detalhes que perpassam a anatomia, subjuga muitos discentes que estão começando os estudos na área (MOORE, 2004).A morfologia estudada na anatomia é um dos maiores obstáculos para os discentes devido a complexidade e grande quantidade de nomes que,comumente,faz com que oensinoseja monótono e desanimador (SALBEGO et al., 2015), sendo a nomenclatura e classificação anatômicas os conteúdos que geram maior dificuldade. Ao serem questionados sobre a dificuldade nestes conteúdos, $66,7 \%$ dos estudantes relataram apresentar maior dificuldade em aprender a nomenclatura anatômica, enquanto 29\% relataram maior dificuldade na classificação e 4,9\% não responderam. Foi observado que $75,4 \%$ dos estudantes considerou que o roteiro contribuiu com a aprendizagem da nomenclatura anatômica, 82,6\% apontou que contribuiu com a aprendizagem da classificação e 69,6\% que contribuiu com ambas. Estes resultados demonstram que é de fundamental importância para a facilitação da aprendizagem que o docente confeccione recursos didáticos para que os estudantes desenvolvam autonomia para estudo independente (DAMASCENO; CÓRIA-SABINI, 2003), transformando os conteúdos e saberes de forma mediadora (LIBÂNEO, 1994). 0 roteiro de aula prática aqui aplicado demonstrou ser eficiente na facilitação da aprendizagem, tendo contribuído para duas das maiores dificuldades referenciadas pelos discentes: nomenclatura e classificação.

Segundo Natário e Santos (2010), uma das maneiras de ampliar o conhecimento é a participação nas monitorias, já que essa tem a finalidade de auxiliar na formação profissional e proporcionar aos estudantes um espaço de aprendizagem, viabilizando o aprofundamento teórico e até mesmo o desenvolvimento de habilidades associadas à atividade docente do monitor. Mesmo com essas competências, a frequência nas monitorias foi considerada baixa;49,3\% dos estudantes afirmaram participar das monitorias somente quando tinham dúvidas, 17,4\% participaram no dia anterior a avaliação e $33,3 \%$ não participaram. Não houve associação entre o grau de dificuldade que os alunos sentiram em aprender a disciplina e a participação nas monitorias (Qui-quadrado $=0,74389$, grau de liberdade $=1, p=0,6894$ ) . Apesar da baixa frequência, quando questionados se o fato de terem que preencher o roteiro aumentou o interesse pela monitoria, $13 \%$ responderam que aumentou MUITO, 58\% que aumentou POUCO e 29\% que não aumentou NADA. Esses dados corroboram com o fato de que os discentes aproveitam pouco a oportunidade das monitorias, mesmo com o laboratório disponível para uso em diversos horários e com a presença de monitores para auxiliar nas dúvidas, revisões e aprendizado (DAMASCENO; CÓRIA-SABINI, 2003).

Acredita-se que a baixa adesão da monitoria nessa 
instituição deva-se a várias razões. 0 fato do laboratório não estar localizado dentro do campus e situar-se longe das residências dos estudantes, associado à preocupação com o início da vida universitária, longe da casa dos pais,são situações que podem desestimular a participação nesta e em outras atividades. Para muitos estudantes, a saída de casa é tida como algo difícil e o grande volume de exigências acadêmicas e administrativas causam sentimentos de estar perdido e pouca motivação. Além disso, essas mudanças despertam em muitos a autonomia de obter o aprendizado, mas em outros, causa desanimo em buscar o conhecimento e oportunidades de aprendizado (TEIXEIRA, 2008). A aprendizagem requer diversas estruturas do educando, seja física, social ou até mesmo psicológica, neste contexto, este processo não pode ser mecânico, sendo, portanto importante que todo o contexto do estudante seja levado em consideração para que não ocorra impactos na construção do conhecimento (CAMPOS, 2010).

Ao longo da história da anatomia, o cadáverfoi um dos principais meios de estudo utilizado para descrever as diversas estruturas do corpo humano (O'MALLEY; SAUNDERS, 2012). Essa ferramenta é considerada fundamental ao processo de ensino-aprendizagem da anatomia humana e à formação profissional daqueles que pretendem trabalhar na área (COSTA; LINS, 2012; SOCIEDADE BRASILEIRA DE ANATOMIA - SBA, [2012]).No entanto, há estudantes que se sentem apreensivos com o uso do cadáver nas aulas práticas (WILLERS, 2013)e relatam sentir aversão ao cheiro de conservantes, dificuldade em identificar estruturas (DISSABANDARA et al., 2015), aumento na ansiedade, náuseas, insônia, preocupação em ver e tocar, além de imagens recorrentes do morto (SNELLING; SAHAI; ELLIS, 2003). Outros problemas associados a esse meio de estudo é a constante redução do número de corpos não reclamados, o que dificulta o ensino dos profissionais da saúde (SOCIEDADE BRASILEIRA DE ANATOMIA - SBA, [2012]).

Nessa perspectiva, o roteiro acabou se tornando uma alternativa de estudo para os discentes, principalmente àqueles que tiveram percepções negativas e que queriam ter menor contato com as peças naturais. Mas durante a pesquisa, todos tiveram as duas ferramentas de estudo à disposição e, ao avaliar o uso de ambas, 71\% consideraram o roteiro complementar ao uso do cadáver no processo de ensino-aprendizagem e outros
$21,6 \%$ disseram que não foi complementar, os demais não responderam. Esses dados reforçam a importância de combinar uma ou mais ferramentas de ensino para auxiliar os discentes, pois a utilização de outros recursos didáticos além do cadáver, tem contribuído com a apredizagem desses(GUY et al., 2015; LONE et al., 2018; PIAZZA; CHASSOT, 2012; RICH; GUY, 2013). De acordo com Souza (2007) a reflexão no processo de atividades ofertadas é de extrema importancia, visto que os recursos didáticos são instrumentos para auxiliar na superação de limites e dificuldades dos educandos, que associado a intervenção pedagógica, se inter-relacionam e se tornam significativos no aprendizado, alcançando o objetivo proposto para o referido conteúdo. É necessário destacar a precisão na seleção dos recursos e materiais didáticos e também na mediação da ação pedagógica, pois, os estudantes estão no processo de aprendizagem e desenvolvimento e precisam de auxilio para atingir o que é proposto.

0 roteiro foi confeccionado utilizando-se imagens retiradas de atlas anatômicos e assim como o cadáver, também apresenta seus pontos negativos. $\mathrm{O}$ atlas e, consequentemente, o roteiroabordam o conteúdo por meio de ilustrações de peças anatômicas reais ou desenhos, mas são de natureza estática e limitada, o que impossibilita uma visualização em várias dimensões, diferentemente do cadáver, que melhora as habilidades do estudantesobre a percepção tridimensional (KLEMT; INFANTOSI, 2000). Isso mostra o quanto é necessário a utilização de diversos recursos didáticos que permita ao educando refletire compreendero conteúdo apresentado, além de estimular sua criatividade e, dessa forma, se apropriar e utilizar desses recursospara a produção de novos conhecimentos a partir dos conhecimentos iniciais (SOUZA, 2007).

Apesar das limitações e de alguns alunos relatarem que parte do conteúdo do roteiro não é apresentada em aula pelo professor, o usodesse material no estudo da anatomia na UFU - campus pontal, foi uma das melhores alternativas de complementação no ensino devido as restrições encontradas no laboratório e ao pouco tempo para ministrar a disciplina, pois, além da fácil manipulação, esta ferramenta trazia assuntos que não poderiam serabordados em aula devido ao tempo disponível. Desse modo, os dicentes eram estimulados a discutirem uns com os outros e a pesquisarem as respostas para 
preencher o roteiro, favorecendo a construção do conhecimento e minimizando os problemas com a carga horária da disciplina, que é um fator que interfere no estudo da anatomia(LIMA; GUEDERT, 2016).

A baixa disponibilidade de cadáveres, recursos e limitações no tempo do currículo fez com que novos métodos fossem incluídos no ensino tradicional da anatomia (HEYLINGS, 2002).De acordo com essas concepções,o roteiro foi elaborado e introduzido nas aulaspara sanar as dificuldades no ensino-aprendizagem. Nessa perspectiva,89,9\% dos estudantes afirmaram que o mesmo contribuiu nesse processo e $7,2 \%$ que não contribuiu, os demais participantes não responderam à questão enão houve diferença significativa entre os gêneros e as modalidades para esses questionamentos. A efetividade do roteiro na facilitação do processo de ensino-aprendizagem pode ser constatada, ainda, pela melhora no desempenho dos estudantes, sendo observada uma redução de $23,5 \%$ de reprovas no ano de 2017 para $15,78 \%$ no ano de 2018 para o curso de licenciatura e manutenção de índices de reprova em torno dos 5\% para estudantes do bacharelado.

A discussão sobre ouso dessa ferramenta em aulas práticas de anatomia humana são escassos na literatura, (MORAES; SCHWINGEL; SILVA JÚNIOR, 2016), contudo, nos estudos realizados, constatou-se que o roteiro é uma experiência positiva e útil nas práticas de dissecção, facilita o estudo em casa e é apreciado pelos alunos como uma ferramenta complementar no estudo da anatomia (DEMARCHI et al, 2014). Segundo Pereira e Xavier (2014), o roteiro proporcionou aos estudantes a interpretação, entendimento e memorização dos termos anatômicos citados durante as aulas e leituras dos livros texto, além de proporcionar o enriquecimento do vocabulário. Outros estudos que fizeram uso dessa ferramenta constataram que essa beneficiou não só os discentes, mas os monitores e o docente (SILVA JÚNIOR et al., 2014), além de contribuir com a otimização do tempo de estudo (OLIVEIRA; COSTA; MITRI, 2014). Apesar de todas essas contribuições, notou-se que cada roteiro apresentava suas particularidades, atendendo as necessidades de cada turma que o utilizava.

\section{CONSIDERAÇÕES FINAIS}

A anatomia é estudada em diversos cursos e os materiais disponíveis geralmente não suprem as demandas da disciplina ou não são os ideais para o estudo de determinadas áreas profissionais. Por esse motivo, observamos a necessidade de que os recursos didáticos atendam as especificidades e demanadas de cada curso e dos discentes que tem como componente curricular esta disciplina. Sendo assim, sugerimos que mais estudos sejam realizados na busca de desenvolver outros métodos de ensino que resultem em maiores condições e possibilidades de aprendizado por parte dos educandos e que cada docente se sensibilize quanto a importância e uso dessas novas ferramentas para intervenção pedagógica no processo de aprendizagem do estudante.

Ao aplicar o roteiro teórico-prático durante a disciplina de Anatomia Humana observamos que os alunos apresentaram um maior envolvimento com o estudo da disciplina, passando a utilizar cada vez mais os materiais e espaços de estudos presentes na universidade, como a biblioteca e as diversas bibliografias disponíveis da área. A avaliação dessa ferramenta mostrou o quanto ela auxilia o professor e os discentes no estudo desse componente curricular,contribuindo com o processo de ensino-aprendizagem ao invés de apenas memorizar o conteúdo. Contudo, destacamos que o cadáver e os demaismateriais didáticos presentes no laboratório também foram essenciais na construção do conhecimento dos envolvidos, portanto, concluímos que o roteiro deve ser utilizado juntamente com outros métodos de ensino para atender de maneira global as necessidades de aprendizado, pois o uso limitado de métodos para ensino não contempla de maneira significativa a construção do conhecimento de todos os estudates.

\section{AGRADECIMENTOS}

Agradecemos à todos os discentes e a Prof. ${ }^{\text {a }}$ Dr. ${ }^{-}$Kátia Gomes FacureGiaretta pela colaboração na pesquisa, e ao Programa de Educação Tutorial (PET Bio Pontal) pelo apoio e suporte no desenvolvimento do roteiro. 


\section{REFERÊNCIAS}

ARRUDA R. M.; SOUSA, C. R. A. Aproveitamento teórico-prático da disciplina anatomia humana do curso de fisioterapia. Revista Brasileira de Educação Médica, Rio de Janeiro, v. 38, n. 1, p. 65-71, jan./mar. 2014. Disponível em: <http://dx.doi. org/10.1590/S0100-55022014000100009>. Acesso em: 02 abr. 2018.

BRAZ, P. R. P. Método didático aplicado ao ensino da anatomia humana. Anuário da Produção Acadêmica Docente, Valinhos, v. 3, n. 4, p. 303-310, dez. 2009. Disponível em: <http:// repositorio.pgs skroton.com.br/bitstre am/123456789/1342/1/Artigo\%2020.pdf>. Acessoem: 04 abr. 2018.

BRUECKNER, J. K.; MACPHERSON, B. R. Benefits from peer teaching in the dental gross anatomy laboratory. European Journal of Dental Education, v. 8, n. 2, p. 72-77, mai. 2004. Disponívelem: <https://doi.org/10.1111/j.1600-0579.2003. 00333.x>. Acesso em: 29 mar. 2018

CAMPOS, D. M. S. Condições psicológicas da aprendizagem: a motivação. In:___. Psicologia da aprendizagem. 38. ed. Petrópolis: Vozes, 2010. p. 108-126.

COSTA, G. B. F.; LINS, C. C. S. A. 0 cadáver no ensino da anatomia humana: uma visão metodológica e bioética. Revista Brasileira de Educação Médica, Rio de Janeiro, v. 36, n. 3, p. 369-373, jul./set. 2012. Disponível em: <http://dx.doi.org/10.1590/ S0100-55022012000500011>. Acesso em: 26 mar. 2018.

DAMASCENO, S. A; CÓRIA-SABINI, M. A. Ensinar e aprender: saberes e práticas de professores de anatomia humana. Revista Psicopedagogia, Pinheiros, v. 20, n. 63, p. 243-254, set./dez. 2003. Disponível em: <http://www.revistapsicopedagogia com.br/detalhes/286/ensinar-e-aprender--saberes-e-praticas-de-professores-de-anatomia-humana>. Acesso em: 03 abr 2018.

DEMARCHI, A. V. et al. A importância do roteiro e suas implicações no aprendizado prático da anatomia. In: CONGRESSO BRASILEIRO DE ANATOMIA, 26, 2014, Curitiba. Anais... Curitiba: [s.n.], 2014. Disponível em: <https://www.researchgate. net/profile/Edivaldo_Silva_Junior/publication/303288293_671_-_Relato_de_Experiencia_de_Monitores_de_Anatomia_Humana_do_Curso_de_Fisioterapia_na_Dissecacao_Cadaverica/links/573b2a6208ae298602e44 ca6/671-Relato-de-Experiencia-de-Monitores-de-Anatomia-Humana-do-Curso-de-Fisioterapia-na-Dissecacao-Cadaverica.pdf>. Acessoem: 03 abr. 2018.

DISSABANDARA, L. O. et al. Role of cadaveric dissections in modern medical curricula: a study on student perceptions. Anatomy \& Cell Biology,Seongnam, v. 48, n. 3, p. 205-212, set. 2015. Disponívelem: <https://doi.org/10.5115/ acb.2015.48.3.205>. Acesso em: 03 abr. 2018.
FALAVIGNA, G. Inovação e multimídia na educação. In: Inovaçoes centradas na multimídia: repercussões no processo de ensino-aprendizagem. 1. ed. Porto Alegre: EDIPUCRS, 2009. p. 31-133.

FRUHSTORFER, B. H. et al. The use of plastinatedprosections for teaching anatomy: the view of medical students on the value of this learning resource.ClinicalAnatomy,v. 24, n. 2, p. 246 252, mar. 2011. Disponível em: <https://doi.org/10.1002/ ca.21107>. Acessoem: 29 mar. 2018.

GHOSH, S. K. Cadaveric dissection as an educational tool for anatomical sciences in the 21st century. AnatomicalSciencesEducation, Hoboken, v. 10, n. 3, p. 286-299, mai./jun. 2017. Disponível em: <https://doi.org/10.1002/ase.1649>. Acessoem: 27 mar. 2018.

GLITTENBERG, C.; BINDER, S. Using 3D computer simulations to enhance ophthalmic training. Ophthalmic and Physiological Optics,v. 26, n. 1, p. 40-49, jan. 2006. Disponívelem: <https://doi.org/10.1111/j.1475-1313.2005.00358.x>. Acessoem: 29 mar. 2018

GUY, R. et al. Less is more: development and evaluation of an interactive e-atlas to support anatomy learning. AnatomicalSciencesEducation, Hoboken, v. 8, n. 2, p. 126-132, mar./abr. 2015. Disponível em: <https://doi.org/10.1002/ase.1461>. Acessoem: 27 mar. 2018.

HEYLINGS, D. J. A. Anatomy 1999-2000: the curriculun, who teaches it and how?.Medical Education, Oxford, v. 36, n. 8, p 702-710, ago. 2002. Disponível em: <https://doi.org/10.1046 /j.1365-2923.2002.01272.x>. Acesso em: 03 abr. 2018.

JOHNSON, E. O.; CHARCHANTI, A. V.; TROUPIS, T. G. Modernization of an anatomy class: from conceptualization to implementation. A case for integrated multimodal-multidisciplinary teaching. Anatomical Sciences Education,Hoboken, v. 5, n. 6 p. 354-366, nov./dez. 2012. Disponívelem: <https://doi. org/10.1002/ase.1296>. Acessoem: 27 mar. 2018.

KHARB, P.; SAMANTA, P. P. Blended learning approach for teaching and learning anatomy: students' and teachers' perspective. Journal of the Anatomical Society of India, v. 65, n. 1, p. 43-47, jun. 2016. Disponível em: <https://doi. org/10.1016/j.jasi.2016.06.001>. Acesso em: 29 mar. 2018.

KLEMT, A.; INFANTOSI, A. F. C. Método de superfície na visualização 3D da dissecação do crânio humano. Revista Brasileira de Engenharia Biomédica, Rio de Janeiro, v. 16, n. 1, p. 21-37, jan./abr. 2000. Disponível em: <http://host-article-ass e ts . s 3 - we b sit e-u s-eas t-1.a mazon aw s.com / rbeb/5889fb815d01231a018b466c/fulltext.pdf $>$. Acesso em: 03 abr. 2018

KRUSE, M. E. L. Anatomia: a ordem do corpo. Revista Brasileira de Enfermagem, Brasília, v. 57, n. 1, p. 79-84, jan./fev. 2004. Disponível em: <http://dx.doi.org/10.1590/S003471672004000100017>. Acesso em: 26 mar. 2018. 
KRYCH, A. J. et al. Reciprocal peer teaching: students teaching students in the gross anatomy laboratory. ClinicalAnatomy,v. 18, n. 4, p. 296-301, mai. 2005. Disponível em: <https://doi. org/10.1002/ca.20090>. Acesso em: 29 mar. 2018.

LIBÂNEO, J. C. O processo de ensino na escola. In: Didática. 1. ed. São Paulo: Cortez, 1994. p. 77-102.

LIMA, P.; GUEDERT, D. G. Estudo da anatomia humana da graduação em fisioterapia na Universidade Regional de Blumenau: uma visão documental e discente. Atos de Pesquisa em Educação, Blumenal, v. 1, n. 11, p. 271-292, jan./abr. 2016. Disponível em: <http://dx.doi.org/10.7867/1809-0354.2016v11n1p271-292>. Acesso em: 02 abr. 2018.

LONE, M. et al. Evaluation of an animation tool developed to supplement dental student study of the cranial nerves. European Journal of Dental Education, v. 22, n. 3, p. 427-437, ago. 2018. Disponívelem: <https://doi.org/10.1111/eje.12321>. Acesso em: 27 mar. 2018.

MASETTO, M. T. A sala de aula - espaço de vida?. In: . Didática: a aula como centro. 4. ed. São Paulo: FTD, 1997. p. $29-40$.

MOORE, K. L.; AGUR, A. M. R. Fundamentos de anatomia clínica. 2. ed. Rio de Janeiro: Guanabara Koogan, 2004.

MORAES, G. N. B.; SCHWINGEL, P. A.; SILVA JÚNIOR, E. D. Uso de roteiros didáticos e modelos anatômicos, alternativos, no ensino-aprendizagem nas aulas práticas de anatomia humana. Revista Ibero-Americana de Estudos em Educação, Araraquara, v. 11, n. 1, p. 223-230, jan./mar. 2016. Disponível em: <https://doi.org/10.21723/riaee.2016.v11.n1.p223>. Acesso em: 03 abr. 2018.

NASCIMENTO, I. Y. M. et al. Confecção de modelos neuroanatômicos, sadios e infectados por parasitas, para o ensino - aprendizagem da neuroanatomia e parasitologia humana. In: CONGRESSO NACIONAL DE EDUCAÇÃO, 1, 2014, Campina Grande. Anais... Campina Grande: Realize, 2014. Disponível em: <http://www.editorarealize.com.br/revistas/conedu/trabalhos/ Modalidade_4datahora_14_07_2014_12_50_05_idinscrito_32 23_68a4980da6ffaa100bf7a94f0a1d714a.pdf> Acesso em: 26 mar. 2018

NATÁRIO, E. G.; SANTOS, A. A. A. Programa de monitores para o ensino superior. Estudos de Psicologia, Campinas, v. 27, n. 3, p. 355-364, jul./set. 2010. Disponível em: <http://dx.doi. org/10.1590/S0103-166X2010000300007>. Acesso em: 02 abr. 2018.

NETTER, F. H. Atlas de anatomia humana. 5. ed. Rio de Janeiro: Elsevier, 2011

O'MALLEY, C. D.; SAUNDERS, J. B. C. M. Os cadernos anatômicos de Leonardo da Vinci. 1. ed. Cotia: Ateliê Editorial; Campinas: Editora Unicamp, 2012.
OLIVEIRA, A. G.; COSTA, A. R. G. F.; MITRI F. F. Criação de novas ferramentas didáticas para apoio ao graduando de odontologia no ensino da anatomia do aparelho estomatognático. In: CONGRESSO BRASILEIRO DE ANATOMIA, 26, 2014, Curitiba. Anais... Curitiba: [s.n.], 2014. Disponível em: <https://www. researchgate.net/profile/Edivaldo_Silva_Junior/publication/303288293_671_-_Relato_de_Experiencia_de_Monitores_de_Anatomia_Humana_do_Curso_de_Fisioterapia_na_Dissecacao_Cadaverica/links/573b2a6208ae298602e44 ca6/671-Relato-de-Experiencia-de-Monitores-de-Anatomia-Humana-do-Curso-de-Fisioterapia-na-Dissecacao-Cadaverica.pdf>. Acessoem: 03 abr. 2018.

PEREIRA, J. A. et al. Effectiveness of using blended learning strategies for teaching and learning human anatomy. Medical Education, Oxford, v. 41, n. 2, p. 189-195, fev. 2007. Disponível em: <https://doi.org/10.1111/j.1365-2929.2006.02672.x> Acesso em: 29 mar. 2018.

PEREIRA, P. L.; XAVIER, G. A. A. Roteiros das origens e significados dos termos anatômicos em aulas de anatomia veterinária. In: CONGRESSO BRASILEIRO DE ANATOMIA, 26, 2014, Curitiba. Anais... Curitiba: [s.n.], 2014. Disponível em: <https://www.researchgate.net/profile/Edivaldo_Silva_Junior/ publication/303288293_671_-_Relato_de_Experiencia_de_ Monitores_de_Anatomia_Humana_do_Curso_de_Fisioterapia_ na_Dissecacao_Cadaverica/links/573b2a6208ae298602e44 ca6/671-Relato-de-Experiencia-de-Monitores-de-Anatomia-Humana-do-Curso-de-Fisioterapia-na-Dissecacao-Cadaverica.pdf>. Acesso em: 03 abr. 2018.

PIAZZA, B. L.; CHASSOT, A. I. Anatomia humana, uma disciplina que causa evasão e exclusão: quando a hipótese principal não se confirma. Ciência em Movimento, Porto Alegre, v. 14, n. 28 p. 45-59. 2012. Disponível em: <http://dx.doi org/10.15602/1983-9480/cmedh.v14n28p45-59>. Acessoem: 29 mar. 2018.

RICH, P.; GUY, R. A “Do-It-Yourself” interactive bone structure module: development and evaluation of an online teaching resource. AnatomicalSciencesEducation, Hoboken, v. 6, n. 2, p. 107-113, mar./abr. 2013. Disponível em: <https://doi org/10.1002/ase.1312>. Acesso em: 27 mar. 2018

SALBEGO, C. et al. Percepções acadêmicas sobre o ensino e a aprendizagem em anatomia humana. Revista Brasileira de Educação Médica, Rio de Janeiro, v. 39, n. 1, p. 23-31, jan./mar 2015. Disponível em: <http://dx.doi.org/10.1590/1981-52712015v39n1e00732014>. Acesso em: 26 mar. 2018.

SILVA JÚNIOR, E. X. et al. Elaboração de roteiros para o ensino-aprendizagem nas aulas práticas das disciplinas de anatomia humana e neuroanatomia. In: CONGRESSO NACIONAL DE EDUCAÇÃO, 1, 2014, Campina Grande. Anais... Campina Grande Realize, 2014. Disponível em: <http://www.editorarealize. com.br/revistas/conedu/trabalhos/Modalidade_1dataho ra_15_07_2014_22_59_18_idinscrito_3160_6ad18d13d81622 ec0227b39630b0b40a.pdf> Acesso em: 03 abr. 2018. 
SIQUEIRA NETO, E. G. B.; FERREIRA, J. R. 0 ensino da anatomia humana no curso de medicina da Universidade Federal de Goiás: avaliação e perspectivas. Arquivos de Ciências da Saúde da UNIPAR,Umuarama, v. 5, n. 1, p. 41-50, jan./abr. 2001. Disponível em: <http://revistas.unipar.br/index.php/saude/article/view/1104/967>. Acesso em: 26 mar. 2018.

SNELLING, J.; SAHAI, A.; ELLIS, H. Attitudes of medical and dental students to dissection. ClinicalAnatomy, v. 16, n. 2, p. 165 172, mar. 2003. Disponível em: <https://doi.org/10.1002/ ca.10113>. Acessoem: 29 mar. 2018

SNELLING, J.; SAHAI, A.; ELLIS, H. Attitudes of medical and dental students to dissection. ClinicalAnatomy, v. 16, n. 2, p. 165172, mar. 2003. Disponível em: <https://doi.org/10.1002/ ca.10113>. Acesso em: 03 abr. 2018.

SOCIEDADE BRASILEIRA DE ANATOMIA. Entendendo a doação de corpos para fins de ensino e pesquisa. [2012]. Disponível em: <http://sbanatomia.org.br/doacao-de-corpos/> Acesso em: 19 jan. 2018.
SOUZA, S. E. 0 uso de recursos didáticos no ensino escolar. Arquivos do Mudi, Maringá, v. 11, p. 110-114, 2007. Suplemento 2. Disponível em: <http://www.dma.ufv.br/downloads/ MAT\%20103/2015-II/slides/Rec\%20Didaticos\%20-\%20 MAT\%20103\%20-\%202015-II.pdf>. Acesso em: 10 jun. 2018.

TEIXEIRA, M. A. P. et al. Adaptação à universidade em jovens calouros. Psicologia Escolar e Educacional, Campinas, v. 12, n. 1, p. 185-202, jun. 2008. Disponível em: <http://dx.doi. org/10.1590/S1413-85572008000100013>. Acesso em: 03 abr. 2018.

TORTORA, G. J.; NIELSEN, M. T. Princípios de anatomia humana. 12. ed. Rio de Janeiro: Guanabara Koogan, 2013.

WILLERS, C. D. A. et al. 0 processo de ensino-aprendizagem nas disciplinas de anatomia e fisiologia humana direcionado a alunos do curso de ciências biológicas. In: ENCONTRO INTERNACIONAL DE PRODUÇÃO CIENTÍFICA, 8, 2013, Maringá. Anais... [Maringá]: Cesumar, 2013. Disponível em: <https://www.unicesumar.edu.br/epcc-2013/wp-content/uploads/sites/82/2016/07/Cyndi_Dietrich_Andrade_e_Willers_02.pdf>. Acesso em: 26 mar. 2018. 